\title{
Relation of aplastic anaemia to use of chloramphenicol eye drops in two international case-control studies
}

\author{
Bengt-Erik Wiholm, Judith Parsells Kelly, David Kaufman, Surapol Issaragrisil, Micha Levy, \\ Theresa Anderson, Samuel Shapiro
}

Medical Pharmacoepidemiology Unit, Department of Clinical

Pharmacology,

Karolinska Institute, Huddinge

University Hospital,

S-141 86 Huddinge,

Sweden

Bengt-Erik Wiholm, associate professor

Slone

Epidemiology Unit, Boston University

School of Medicine,

Brookline,

MA 02146, USA

Judith Parsells Kelly, epidemiologist

David Kaufman, assistant director

Theresa Anderson, study coordinator Samuel Shapiro, director

Mahidol University, Bangkok, Thailand Surapol Issaragrisil, professor

Hadassah

University Hospital, Jerusalem, Israel

Micha Levy,

professor

Correspondence to: Professor Wiholm, Medical Products Agency, PO Box 26, S-75103 Uppsala,

Sweden

beje.wiholm@mpa.se

BMJ 1998;316:666
Although the use of chloramphenicol eye drops is thought to cause aplastic anaemia, ${ }^{1}$ this side effect has not been studied critically. We examined the use of ocular chloramphenicol in two population based case-control studies conducted with the same methods. $^{23}$

\section{Subjects, methods, and results}

The data from the international granulocytosis and aplastic anaemia study were collected over varying times from 1980 to 1986 in Israel and in Ulm and Berlin (Germany), Milan (Italy), Budapest (Hungary), Sofia (Bulgaria), and Stockholm and Uppsala (Sweden); the total base population was about 19 million. ${ }^{2}$ Data collection continued independently in Sweden until 1992. The Thai study was conducted from 1989 to 1994 in Bangkok and from 1990 to 1994 in Khonkaen and Songkla; the total population was about 21 million. $^{3}$

In both studies patients with aplastic anaemia were identified by regular telephone contacts with all hospitals in the study regions. Cases were patients whose peripheral blood counts and bone marrow histology met accepted diagnostic criteria ${ }^{2}$; each diagnosis was confirmed by a panel of haematologists blinded to drug exposures. Controls matched for age and sex were selected from among patients admitted to the same hospitals within 3 months for reasons judged to be independent of antecedent drug use-for example acute trauma. ${ }^{2}$ Data on exposure to chloramphenicol were obtained by interview and included detailed histories of all drug use (including topical drugs) in the 6 months before admission; lists of indications for use and trade names of drugs of particular interest were used to help recall. The time of onset of aplastic anaemia could not be determined with precision because some symptoms, such as fatigue, are vague. Therefore, any use in the 5 month period from 6 months to 1 month before admission was judged to be aetiologically relevant.

There were 426 cases and 3118 controls. None of the cases had used chloramphenicol eye drops, but seven of the controls had used them (table $\left.{ }^{1}\right)$. The upper

Exposure to chloramphenicol in seven controls

\begin{tabular}{llcccccc} 
Control No & Region & $\begin{array}{c}\text { Age } \\
\text { (years) }\end{array}$ & Sex & Route & Frequency & $\begin{array}{c}\text { Last use } \\
\text { before } \\
\text { admission }\end{array}$ & Duration \\
\hline 1 & Thailand & 9 & M & Ocular & Daily & 3rd month & 2 days \\
\hline 2 & Thailand & 21 & M & Ocular & Daily & 4th month & 1 day \\
\hline 3 & & & & Oral & Daily & 2nd month & 3 days \\
\hline 4 & Thailand & 63 & F & Ocular & Daily & 5th month & 5 days \\
\hline 5 & Thailand & 29 & F & Ocular & Daily & 3rd month & 3 days \\
\hline 6 & Israel & 66 & M & Ocular & Daily & 1 day & 2 months \\
\hline 7 & Sweden & 34 & F & Ocular & Occasional & 1 day & 5 years \\
\hline & Israel & 19 & M & Ocular & Daily & 3rd month & 3 days \\
\hline
\end{tabular}

$95 \%$ confidence limit for a relative risk of zero ${ }^{4}$ was 5.1 . Five of the exposed controls $(0.2 \%)$ had been exposed for $\geqslant 3$ days.

\section{Comment}

We conducted two population based studies using virtually identical protocols representing about 185 million person years of observation in industrialised and developing countries. Among more than 400 cases of aplastic anaemia there was no use of chloramphenicol eye drops. Although there was no evidence of an association the prevalence of use among the controls was low, and the possibility of some increase in risk cannot be excluded.

Bias is unlikely to account for the findings, since associations were found in these data for several other previously suspected drugs. ${ }^{23}$ Information bias, in particular, is improbable: chloramphenicol has long been suspected as a cause of aplastic anaemia, and the likely result of any such bias would have been an overestimation of the risk. Definition of the appropriate window of exposure for drug induced aplastic anaemia is more problematic. Because of the insidious onset of aplastic anaemia we excluded any drug use that started in the month before admission.

Our data provide no support to the claim that chloramphenicol eye drops increase the risk of aplastic anaemia. ${ }^{1}$ The literature lends only weak support for causality, being based on a few single case reports that are often inadequately investigated and have other possible causes. The recommendation that chloramphenicol eye drops should be avoided because of an increased risk of aplastic anaemia ${ }^{1}$ is not well founded.

Contributors: BEW had the idea for the present analysis, initiated the work and drafted the paper. JPK did the combined analysis of the two studies. The international granulocytosis and aplastic anaemia study was designed by SS, ML, who was the chairman of the study, and DK. The Thai study was designed by SI, who was the principal investigator, DK and SS. Both studies were coordinated and quality controlled by TA. All authors contributed ideas and helped with the final writing of the paper. BEW and DK are guarantors of the work.

Funding: The present analysis had no external funding. The study was primarily sponsored by Hoechst, Frankfurt, Germany, with additional support from Apoteksbolaget, Sweden, and the governments of Hungary and Bulgaria. The Thai study was sponsored by grant number R01-HL35068 from the US National Heart Lung and Blood Institute.

1 Doona M, Walsh JB. Use of chloramphenicol as ocular eye medication: time to cry halt? $B M J 1995 ; 310: 1217-8$

2 Kaufman DW, Kelly JP, Levy M, Shapiro S. The drug etiology of agranulocytosis and aplastic anemia. New York: Oxford University Press, 1991.

3 Issaragrisil S, Kaufman DW, Anderson T, Chansung K, Thamprasit T, Sirijiachai J, et al. Low drug attributability of aplastic anemia in Thailand. Blood 1997;89:4034-9.

4 Thomas DG. Exact confidence limits for the odds ratio in a 2x2 table. Appl Stat 1971;20:105-10.

(Accepted 21 July 1997) 